\title{
La espontaneidad del deber en Kant
}

The sponteneity of the duty in Kant

\author{
RAFAEL REYNA FORTES \\ Universidad de Navarra (España)
}

recibido: 08.06 .2015

aceptado: 25.07 .2015

\section{RESUMEN}

Este trabajo intenta hacer una propuesta, basándose en los trabajos del Kant precrítico, para entender uno de los puntos más importantes en la filosofía crítica: el deber: El principal objetivo de este trabajo es mostrar la relación entre este concepto y la noción de espontaneidad, entendida como una nota de los conceptos intelectuales

PALABRAS CLAVE

DEBER, ESPONTANEIDAD, SENTIMIENTO, INTELECTO

\begin{abstract}
This paper tries to make a proposal, based in the precritical works of Kant, to understand one of the most importants points of the critical philosophy: the duty. The main aim of this work is to show the relationship between that concept and the notion of spontainety as a note of the intelectual concepts.

KEY WORDS

DUTY, SPONTANEITY, FEELING, INTELLECT
\end{abstract}

Claridades. Revista de filosofía 7 (2015), pp. 45-58

ISSN: 1889-6855 ISSN-e: 1989-3787 Dl.: PM 1131-2009

Asociación para la promoción de la Filosofía y la Cultura (FICUM) 


\section{INTRODUCCIÓN}

LA HISTORIA DE LA FILOSOFÍA PUEDE VERSE, hasta cierto punto, como una reformulación de las mismas cuestiones. Sin respuestas y con multitud de objetores, toda tradición filosófica que ha intentado responder a tales interrogantes ha sido censurada, ya por sus mismos representantes, ya por verdaderos disidentes. En cualquier caso, existe la posibilidad de hablar, incluso después de la posmodernidad, de una filosofía; y ésta, perenne, está formada por todo el conjunto de autores que han traído luces a las preguntas antedichas. Tales pensadores, si bien es verdad que han aportado claridad y han continuado lo que permanecía inacabado, han puesto los cimientos de nuevos proyectos. Éstos acaban por auto-censurarse en la mayoría de los casos, pero si hay una tradición de filósofos que se ha caracterizado por la creación de proyectos filosóficos, esa ha sido la modernidad. Y de entre todos los que la conforman hay uno del que puede decirse, por su influencia y profundidad, que encarna a la perfección el ideal moderno: Immanuel Kant.

En efecto, si se entiende la filosofía como se ha dicho más arriba, como una permanente reformulación, el pensamiento de Kant no sólo es revolucionario, sino que además fue una revolución contra él mismo. Y así es, el itinerario intelectual de Kant no puede verse, con una mirada atenta, como un camino llano hacia el idealismo trascendental. Todo lo contrario, los escarpados planteamientos, los callejones sin salida, las caídas, decepciones y renuncias tienen un claro protagonismo en el proceso evolutivo de Kant. Pues bien, todo este recorrido marcó, por así decir, el punto de llegada del alemán hasta tal punto que hoy resulta necesario prestar atención a los escritos precríticos para iluminar aspectos de la doctrina ya crítica.

Pues bien, el objetivo de este trabajo es desvelar los cimientos históricos sobre los que se sustenta uno de los aspectos más reconocidos de la filosofía de Kant: el deber moral. No es que por la gnoseología planteada en $K r V$ el alemán no mereciera ya un lugar privilegiado entre los grandes de la filosofía, sino que su doctrina moral, apoyada en su doctrina especulativa, ha tenido un alcance y una profundidad notables. Para tal objetivo voy a tomar dos textos ${ }^{1}$ del Kant precrítico y los pondré en relación,

1 KANT, I., "Sobre la nitidez de los principios de la teología natural y la moral", trad. de Roberto Torretti, Diálogos, 27, (1974), pp. 57-87, y KANT, I., Sobre la forma y los principios

Claridades. Revista de filosofía 7 (2015) 
en la medida en que se pueda, con un aspecto del deber que se destaca en la Fundamentación de la metafísica de las costumbres: la espontaneidad con la que se plantea el deber.

\section{LA INVESTIGACIÓN SOBRE LA CLARIDAD DE \\ LOS PRINCIPIOS DE TEOLOGÍA NATURAL Y DE MORAL}

La primera de las obras a la que me voy a referir es a la Untersuchung. Ésta vio la luz en 1763 con ocasión de un concurso que organizaba la Real Academia de las Ciencias de Berlin. El tema de éste trataba sobre la claridad de los conceptos metafísicos. Y esta, como es obvio, será el objetivo: plantear qué tipo de certeza poseen los conceptos metafísicos. Pero, además, Kant desarrolla aquí un método para que la metafísica pueda gozar los éxitos que tiene la física newtoniana. Hay que recordar, para captar la importancia de esta obra, que los años en los que se escribe, a principio de los 60, son años tremendamente convulsos. Se acaban de publicar Beweisgrund y Negative Grössen, dos obras de gran importancia para la filosofía de su tiempo. Podría decirse que éstas constituyen el símbolo de la ruptura de Kant con la doctrina racionalista en la que se había educado. El método que Kant diseñará en la Untersuchung será un método para una metafísica, por tanto, que no es la racionalista y que pondrá sus esperanzas en el rigor de Newton. En efecto, si se siguen tales reglas, el término de toda investigación no será otro que la verdad. Este es el proyecto de la Untersuchung.

Si se atiende, en cambio, al título completo de la obra en cuestión se puede ver lo siguiente: se trata de un estudio de la claridad (Deutlichkeit) de los principios de teología natural y de moral. Por tanto, si bien es verdad que Kant desarrolla un método para la metafísica en este obra, no es menos cierto que el objetivo que se marca con el título es el de explicar por qué la teología natural y la moral gozan de la claridad de la ciencia de Newton. Para explicar bien esto, compararé en qué consiste la certeza de los conceptos metafísicos y la de los de la moral.

del mundo sensible y del inteligible, trad. Ramón Ceñal, Consejo superior de investigaciones científicas, Madrid, 1961. 


\section{II.1 LA CLARIDAD DE LOS CONCEPTOS METAFÍSICOS Y MORALES}

En la Untersuchung Kant procede a la explicación de la claridad de tales conceptos comparándolos con los de la matemática. Pues bien, el texto dice lo siguiente:

"A cualquier concepto universal se puede llegar de dos maneras, o bien por enlace arbitrario de conceptos, o bien por abstracción de la representación cognitiva que el análisis ha hecho nítida. La matemática concibe sus definiciones de la primera manera [...] Muy distinto es el caso de las definiciones en filosofía. Aquí el concepto de una cosa está ya dado, pero de modo confuso o insuficientemente determinado"2

Hay, pues, conceptos matemáticos, de un lado, y filosóficos (o metafísicos), de otro. Los primeros surgen de sintetizar conceptos. Por ejemplo, al sintetizar los elementos de un triángulo (tres líneas, 3 ángulos que suman $180^{\circ}$, etc.) obtengo una definición bajo la cual se recogen todos los triángulos posibles. Es más, sólo hay triángulos en la medida que hay cosas que se acoplan a la definición dada. En cambio, los conceptos metafísicos están ya dados y hay que esclarecerlos a través del análisis. Por usar el propio ejemplo de Kant: el concepto de tiempo. Éste nunca acaba de definirse, pero está ya dado. Todos sabemos qué es el tiempo, pero ninguno sabe dar una definición de éste.

El método de Kant consistirá en tomar una nota del concepto dado, metafísico, e ir tomando de éste lo que pueda resultar de base firme para construir una metafísica. Independientemente del rendimiento que tenga, hay que partir de unas pocas premisas e ir construyendo sobre éstas. Kant añade a este respecto otras diferencias entre matemática y filosofía que no son de tanto interés a este trabajo. Por lo que a éste atañe, baste decir que la claridad de los conceptos matemáticos nace de una necesidad analítica. Es decir, que un triángulo, por ejemplo, tiene tres rayas, lo sabemos porque en la definición de triángulo está el tener tres rayas como formando algo esencial de éste. En cambio, en el concepto de tiempo, no aparece nada esencial que se pueda extrapolar de éste. En esta línea, Kant distingue dos tipos de certeza:

"Uno tiene certeza en cuanto sabe que es imposible que un conocimiento sea falso. El grado de esta certeza, considerado objetivamente, depende de la suficiencia en lo que

${ }^{2}$ Untersuchung, pp. 60-61.

Claridades. Revista de filosofía 7 (2015) 
toca a las características de la necesidad de una verdad; pero si se lo considera subjetivamente es tanto mayor cuanto más intuición contenga el conocimiento de esa necesidad"3

Hay, pues, una certeza que surge, como se puede ver, de la propia definición del objeto y otra que surge de la intuición que se tenga de éste. $\mathrm{O}$ sea, hay una certeza objetiva, que atañe al objeto, y que se desprende de la igualdad entre predicado y sujeto y otra que surge subjetiva que depende de la forma en la que el objeto es dado al sujeto. La certeza de la metafísica es del segundo tipo, pues sus conceptos son dados de manera oscura. El error surge de negar (o afirmar) un atributo a alguna cosa que si tiene (o no) tal atributo. La matemática, por tanto, no está abierta al error, pues ella pone la definición de su objeto. Por su parte, la metafísi$\mathrm{ca}$, dado que no tiene nunca su objeto completamente puede afirmar o negar un atributo de él inconvenientemente.

Interesa para este trabajo remarcar bien lo que significa esa certeza objetiva. Ésta consiste en poder afirmar $X$ de un objeto que se ha definido ya como $X-Y-Z$. Así, el objeto es coetáneo, simultáneo, con la definición de éste. El triángulo, por ejemplo, es el dibujado por el geómetra, nada más ni nada menos. La mirada, por tanto, del geómetra tiene ante sí todo el objeto en toda su simplicidad o complejidad. Por su parte, la certeza metafísica consiste en tener un objeto dado (no se sabe cómo) cuyas notas desconozco. Para predicar de él algo, necesitaría un análisis de lo más inmediato de éste y partir de ahí. En suma, la certeza objetiva surge de la representación inmediata del objeto y la subjetiva de la predicación de algo de un objeto sin tenerlo entero a la vista.

Así, para llegar a formular un método para la metafísica hay que llegar a verdades indudables acerca de los objetos dados e ir construyendo desde éstas. Por tanto, la labor prescrita a la metafísica, por el momento, no es otra que la de analizar hasta alcanzar conceptos o principios fundamentales que sustenten toda su actividad posterior. ¿Cuál será, pues, el término del análisis metafísico? Conceptos, en efecto, inanalizables. Sin embargo, un concepto puede ser tal cosa por dos motivos: por su claridad, o por su oscuridad. No se puede dudar de que un triángulo tenga tres rayas, por ejemplo. Pero sí se puede dudar de que al mover el brazo

${ }^{3}$ Untersuchung, p. 77. 
lo hago, o bien por un acto de la voluntad, o bien por una moción física ${ }^{4}$. Los conceptos que pueden fundar una ciencia son los inanalizables claros, pero los que cancelan la posibilidad de una ciencia son los inanalizables oscuros.

Para la metafísica, como era de esperar en Kant, los conceptos siempre son inanalizables oscuros. No ocurre así con la moral y la teología natural. En el caso de ésta última, se debe a que sólo puede haber una causa primera y, por tanto, todo lo que se pueda desprender de ella sólo le compete a ella en cuanto tal. Sin embargo, lo que interesa para este trabajo es lo que ocurre con los conceptos de moral.

Para explicar los fundamentos de la moral Kant establece las siguientes distinciones:

\begin{abstract}
"Uno debe hacer algo esto o aquello, y evitar esto otro. Todo deber expresa una necesidad de la acción y admite dos acepciones. O bien, yo debo hacer algo (como medio), si quiero otra cosa (como fin); o bien yo debo inmediatamente hacer algo (como fin) y hacerlo de veras. La primera puede llamarse la necesidad de los medios (necessitas problemática), la segunda, la necesidad de los fines (necessitas legalis)." 5
\end{abstract}

Por establecer una analogía con lo ya dicho pueden verse los fines como esos conceptos inanalizables claros, que sirven para fundar una metafísica o, en este caso, una moral. Se trata de un fin que, una vez puesto, hace que el hombre despliegue una serie de acciones que son una tentativa para alcanzar ese fin, deberes. Estos fines que se me presentan de manera inmediata hacen que un individuo, por así decir, oriente hacia ellos toda su vida práctica. Los deberes, pues, que plantean son deberes necesarios para todo individuo. En cambio, los deberes que plantean que plantean la necesidad de los medios son más imperfectos. Así, los fines que son queridos por sí actúan como los conceptos inanalizables claros, es decir, permite construir una ética, por así decir, a priori, independientemente de los fines que uno pueda elegir ${ }^{6}$.

\footnotetext{
${ }^{4}$ Este ejemplo es puesto en Los Sueños de un Visionario y es usado para negar la posibilidad de una psicología racional. No aparece en la Untersuchung, pero es aclaratorio.

${ }^{5}$ Untersuchung, p. 84.

6 "Por ejemplo, debo promover la máxima perfección global o debo actuar de acuerdo con la voluntad de Dios; cualquiera de estas dos proposiciones, si se le subordina toda la filosofía práctica y ha de servir por ende como normal y fundamento de la obligación, tiene que prescribir la acción como inmediatamente necesaria y no bajo la condición de
} 
La simplicidad con la que se plantea la acción buena sirve de cimiento de un sistema de acciones morales buenas en sí mismas. O sea, al darse un bien que se debe seguir en todos los casos, se da también la posibilidad de orientar todo el actuar humano hacia dicho fin de modo categórico, no problemático. Ahora bien, ¿̇en qué consiste esta simplicidad del concepto de bien? ¿Es algo añadido a la representación de otra cosa o es algo independiente de toda representación? La respuesta está muy clara en el texto y resulta llamativo atender al lugar donde sitúa Kant la captación de dicho bien:

\begin{abstract}
"Sólo en nuestro tiempos se ha empezado a comprender que la facultad de representarse la verdad es el conocimiento pero la facultad de sentir el bien es el sentimiento y que ambas no deben confundirse. [...] Hay un sentimiento inanalizable de lo bueno (éste no se halla nunca en una cosa por sí sola, sino siempre relativamente a un ser sensitivo). [...] Y es un efecto inmediato de la conciencia del sentimiento de placer que acompaña a la representación"7
\end{abstract}

El sentimiento, por tanto, que acompaña a la representación de una cosa es lo que funda su bondad o maldad. Es curioso que sea precisamente el sentimiento el que dé lugar a este añadido de la representación, puesto que en su filosofía práctica, Kant distará mucho de esta postura. En cualquier caso, resulta de interés para este trabajo señalar que sea lo que fuere, es un concepto inanalizable claro. Inanalizable porque impide un análisis más profundo; y claro porque, en el caso de algunos principios, estos se presentan como buenos para todos los casos. Así, si se enlaza esto último con lo dicho acerca de la certeza objetiva, puede verse lo siguiente: la certeza subjetiva surgía de la presentación del objeto, por así decir, completo ante el cognoscente. De este modo, no había nada en el objeto que el sujeto no tuviese ante sí. A la hora de juzgar acerca del objeto habría que comparar una nota de éste con el mismo objeto y, de esta manera, tendría lugar un juicio con certeza subjetiva. En esta línea, al ser representado un principio moral supremo al sujeto, éste puede extraer de él todo el conjunto de premisas que determinen su actuar.

La certeza objetiva, pues, de la representación de lo bueno surge de la captación de dicho principio puro. Esta comprensión del principio está

un cierto fin. $\mathrm{Y}$ aquí hallamos que tal normal suprema inmediata de toda obligación tiene que ser absolutamente indemostrable" Ibidem, p. 85.

${ }^{7}$ Ibidem, p. 86. 
fundada en el sentimiento ${ }^{8} \mathrm{y}$, sin embargo, señala Kant que "tiene que ser posible alcanzar el máximo grado de evidencia filosófica en lo que concierne a los primeros principios de la moralidad". . Por tanto, puede desprenderse que "el objeto moral", dado que es aprehendido con la mayor evidencia filosófica, que es la certeza subjetiva, tiene que comparecer por completo ante el sujeto.

\section{EL COMIENZO DEL CRITICISMO EN LA DISSERTATIO}

La Untersuchung, como se dijo, se escribió en 1763. Las obras posteriores de Kant, Sueños de un Visionario (1766) y Sobre el primer fundamento de la distinción de regiones en el espacio (1768), serán capitales para enderezar el dogmatismo del alemán hacia el criticismo por el que es conocido. Durante estos años tiene lugar uno de los grandes hitos de la filosofía crítica: la gran luz del 69. La controversia acerca del sentido, el alcance y los presupuestos de este evento está servida ${ }^{10}$. En cualquier caso, parece haber consenso en que la obra inmediatamente posterior al año de la gran luz es una de las más importantes para entender la filosofía crítica. Esta obra es la Dissertatio. El título completo de la obra es "Disertación acerca de las formas y principios del mundo sensible y del inteligible".

Por diversos avatares de carácter gnoseológico y metafísico, Kant estima oportuno desarrollar una filosofía propedéutica que sirva de método para la metafísica. Es decir, Kant trata de elaborar un método que conduzca a la metafísica a ser una ciencia como de Newton. El camino para este proyecto tiene su punto de partida en la división de facultades cognoscitivas y, correlativamente, también de sus objetos. Y esto resulta de gran interés si se ve desde lo dicho en la Untersuchung, pues allí se dijo que según como se den los objetos, así será la certeza de los razonamientos que de ellos se puedan hacer. Y no sólo eso, se habló de unos con-

\footnotetext{
${ }^{8}$ En este punto Kant refrenda la exposición de Hutcheson, al que criticara en la filosofía práctica ya crítica en Ibidem, p 86.

${ }^{9}$ Ibidem, p. 86.

${ }^{10}$ Hay mucha diversidad de enfoques respecto a este asunto. Algunas de las interpretaciones que considero de mayor interés son: 1) FALGUERAS, I., Perplejidad y filosofía trascendental en Kant, Cuadernos de Anuario filosófico, Serie universitaria $\mathrm{n}^{\mathrm{0}} 71$. Servicio de Publicaciones de la Universidad de Navarra, Pamplona, 1999. 2) Tonelli, G., "Die Umwälzung von 1769 bei Kant” en: Kant Studien, 54, (1963), p. 369-375. 3) VILLACAÑAS, J.L., La formación de la Crítica de la razón pura, Universidad de Valencia, Valencia, 1980.
}

Claridades. Revista de filosofía 7 (2015) 
ceptos que son inanalizables y claros que fundan toda la ciencia matemática o moral. La reflexión hacia el conocimiento lleva a Kant a trasladar la problemática al conocimiento y, por tanto, a distinguir entre tipos de objetos $^{11}$. Esto supone que, en adelante, el que el objeto se presente al sujeto de modo claro y distinto ya no es fundamento de certeza, sino que además es necesario remitir a la facultad por la que se da el objeto. Esto se debe a que, según la potencia de la que provenga, un objeto tendrá unas cualidades o tendrá otras; tendrá un tipo de objetividad o tendrá otra; etc.

Así pues, como el título parece indicar, el conocimiento en general se divide en dos facultades: sensibilidad e intelecto:

"La sensibilidad es la receptividad de un sujeto, por la que es posible que el estado representativo del mismo sea afectado de determinada manera por la presencia de algún objeto. La inteligencia (racionalidad) es la facultad de un sujeto, por la cual es capaz de representarse lo que por razón de su condición no puede penetrar en los sentidos. [...] El objeto de la sensibilidad es lo sensible; y lo que no contiene sino lo que sólo puede ser conocido por la inteligencia, es lo inteligible"12

Ambas facultades, inteligencia y sensibilidad, pues, tienen sus objetos correspondientes. La sensibilidad es la capacidad por la que un sujeto es afectado por un objeto, en cambio, la inteligencia es definida negativamente. Podría ser definida esta última del siguiente modo: es la capacidad por la que un sujeto es capaz de representarse un objeto sin que haya tal afección.

Según lo dicho, un objeto inmuta a la sensibilidad y el sujeto, de este modo, se representa tal objeto. Por su parte, la inteligencia no necesita de ésta afección y se presenta al objeto por sí mismo. Un poco más adelante señala Kant que "lo pensado sensiblemente es representación de las cosas como ellas se nos aparecen, lo pensado intelectualmente, de las cosas como ellas son" ${ }^{\prime \prime}$. Esto quiere decir, que el objeto representado por la sensibilidad, de alguna manera, está también mediado por ella ${ }^{14}$. Por otro lado, el

${ }^{11}$ Cfr. Llano, A., Fenómeno y Trascendencia en Kant, $2^{\text {a }}$ ed., EUNSA, Pamplona, 1973, p. 41.

${ }^{12}$ Dissertatio, \3, p. 10.

13 Ibidem, $\int 4$, p. 10.

14 Esta idea es mencionada por Wolfgang en: CARL, W., Der schweigende Kant. Die Entwürfe zu einer Deduktion der Kategorien vor 1781, Vandehoeck \& Ruprecht, Göttingen, 1989, pp. 18-19. 
objeto de la inteligencia es totalmente puesto por ella, es representado de manera espontánea.

Sin embargo, aunque resulte paradójico, que la índole del sujeto medie en la representación del objeto no resta objetividad a la representación y así lo afirma Kant en $\int 7^{15}$. Quiere esto decir que no hay que entender lo dado por la inteligencia desde la sensibilidad y viceversa. Ahora bien, cuando se señaló el título de la obra se mencionaba la distinción, no de facultades, sino de mundos: el sensible y el inteligible. Si llevamos lo dicho a esto se puede lo siguiente: un mundo está referido a todo lo que el sujeto puede captar y otro, el intelectual, está abierto a todo lo demás; es decir, a todo objeto en general. Aquí es donde se ve la diferencia entre mundo sensible e inteligible. Para aclarar esto, la propia Dissertatio resulta confusa y resulta conveniente mostrar una reflexión del Handschriftlicher Nachlaß kantiano:

"Un todo sintético es aquel cuya síntesis se fundamenta en la posibilidad de las partes, las cuales pueden ser pensadas con anterioridad a la síntesis. Un todo analítico es aquel en el que la posibilidad de las partes presupone la síntesis total. Espacio y tiempo son todos analíticos; los cuerpos son todos sintéticos; los todos sintéticos son compuestos de sustancias, los todos analíticos ni de sustancia ni de accidentes sino de todos de posibles relaciones" 16

El todo sintético es aquel que se funda en la posibilidad de las partes. Por su parte, el analítico que presupone la síntesis de tales partes. No considero necesario demorarme demasiado en la explicación del texto, pero baste decir que el todo analítico es el que la síntesis ya está dada. Esta síntesis se refiere a que el todo analítico ya es uno con independencia de aquello que sintetice. En cambio, el todo sintético es aquel que unifica todo aquello que le es dado. El analítico es descrito por el espacio y el tiempo, leyes de la sensibilidad que, como se vio, es la capacidad de ser afectado por objetos ${ }^{17}$. El todo sintético se funda en los objetos y

\footnotetext{
15 "Por lo dicho se ve que se declara mal lo sensible [diciendo que es] lo conocido confusamente, y lo intelectual [diciendo] que es aquello cuyo conocimiento es distinto" Dissertatio, $\int 7$, p. 12.

16 Reflexionen 3789, Ak. 17, p. 293. La traducción aquí es de Villacañas.

${ }^{17}$ Esta capacidad está, por cierto, situada en el cuerpo. Esta tesis se encuentra de manera implícita en la Dissertatio, pero de modo explícito en Del primer fundamento de la primera diferencia de regiones en el espacio del año 68.
} 
desde ellos despliega la síntesis que los recoge y forma un único todo. Las leyes que lo conforman son las de identidad y no contradicción.

Dicho esto, aquí aparece una cuestión central para la Dissertatio: o se supone que hay objetos cuyas notas no obedecen a la síntesis de las leyes de identidad o no contradicción, o se debe admitir entonces que el objeto del conocimiento intelectual, que forma un todo sintético coincide con lo real. Si esto es así, toman sentido otros pasajes de la Dissertatio donde se dice que lo que no puede ser representado por la inteligencia, tampoco puede ser representado por la sensibilidad, pero no al revés ${ }^{18}$.

El alcance, pues, de la identificación de los todos analítico y sintético con los mundos sensible e inteligible, respectivamente, permite decir que el mundo inteligible describe lo real; y el sensible, las apariencias.

Este recorrido por la Dissertatio permite ahora formular la siguiente pregunta: Los conceptos de la moralidad, si en Untersuchung venían del sentimiento, ahora ¿de dónde vienen? O, más bien, ¿Qué lugar tienen los conceptos morales en la nueva gnoseología kantiana? No pueden estar sólo en lo sentimientos (relativos al cuerpo y a la sensibilidad) y no estar en el intelecto. Dichos conceptos tienen, a fortiori, que adquirir un carácter intelectual. Pues bien, tales conceptos no sólo adquirirán tal carácter, sino que pasarán a desvincularse de la sensibilidad. En adelante ya no serán sentimientos, sino conceptos o ideas puros. Así se afirma en el siguiente texto:

"Sin embargo, cada uno de estos dos conocimientos [sensible e inteligible] conserva el signo de su origen, de manera que el primero, por muy distinto que sea, por razón de su origen siempre es llamado sensible, y el segundo, por muy confuso que sea, siempre permanece intelectual, cuales son, por ejemplo, los conceptos morales conocidos, no por experiencia, sino por el mismo intelecto puro". 19

En efecto, en la Dissertatio los conceptos morales han perdido su carácter sensible y han ganado un carácter intelectual. De este modo, lo que se ha atribuido al conocimiento intelectual puede ahora atribuirse a los conceptos de moralidad:

1) Los conceptos intelectuales se dijeron que eran espontáneos, es decir, que se presentan al sujeto directamente, no cabe duda acerca de ellos precisamente por esto. En cambio, si el concepto es sensible, dado que la

${ }^{18}$ Dissertatio, $\int 1$, pp. $5-6$.
${ }^{19}$ Ibidem, $\int 7$, p. 13.

Claridades. Revista de filosofía 7 (2015) 
sensibilidad tiene su origen en el sujeto es dependiente de él y, por tanto, no se puede "universalizar". Quiero decir con esto que no es un concepto válido para todos los casos.

2) Los conceptos intelectuales son independientes de la experiencia $y$, por tanto, lo que había de relativo a lo contingente o a la corporalidad desaparece ahora. El sujeto, que es principio de las apariencias deja de mediar en la compresión de conceptos morales (como en el caso del sentimiento) y pasa a un conocimiento de certeza objetiva de tales conceptos.

\title{
IV. LA FILOSOFÍA MORAL CRÍTICA Y SU SEMEJANZAS CON
} LA NUEVA DETERMINACIÓN DE LOS CONCEPTOS MORALES

Para cerrar esta exposición voy a usar la traducción del curso que Kant impartió en los años $1784-1785^{20}$. En las lecciones de ética del Kant ya crítico prevalece lo dicho en la Dissertatio acerca de la situación de la moralidad en la inteligencia:

\begin{abstract}
"Ahora bien, aquellos principios que deben ser válidos universal y necesariamente ni pueden ser deducidos de la experiencia, sino de la razón pura. En efecto, la ley moral encierra una necesidad categórica y no una necesidad constituida a partir de la experiencia. Toda regla necesaria ha de ser establecida a priori; se trata por tanto de principios intelectuales"21
\end{abstract}

Los conceptos de moralidad son caracterizados por oposición a los de la experiencia. Los primeros son universales y los segundos impiden la universalización. En este punto resulta de interés remitirse a la universabilidad de los principios de moralidad. Si son universalizables es que no son empíricos y si no son empíricos, son intelectuales. Es decir, dado que los conceptos intelectuales se distinguen por completo de los empíricos, que no son morales, se puede entender el criterio de la universabilidad con un carácter bien distinto. No se trata de que poder universalizar la máxima de la acción por que las consecuencias que se seguirían fueran a ser positivas, sino que, porque son universalizables, son intelectuales y, por tanto morales.

\footnotetext{
${ }^{20}$ Este curso aparece en el tomo XXVII. 1 de las Obras completas de Kant

${ }^{21} \mathrm{~K} A \mathrm{NT}, \mathrm{I} .$, Lecciones de ética, trad. Roberto Rodríguez Aramayo y Concha Roldán Panadero, Editorial Crítica, Barcelona, 1988, p. 51.
}

Claridades. Revista de filosofía 7 (2015) 
Desde esta visión puede señalarse que la caracterización, dada más arriba, por la que se decía que los conceptos intelectuales se distinguen de los empíricos, sirve para delimitar no sólo un carácter distinto de la universabilidad de las máximas morales, sino que permite ver la génesis histórica de tal criterio. Y junto con esta génesis, pueden observarse los motivos gnoseológicos que hace válido dicho criterio.

\section{CONCLUSIÓN}

Para concluir con este trabajo me gustaría señalar los siguientes puntos:

1. Se ha mostrado como desde la década de 1760-70 el pensamiento de Kant ha sufrido un giro drástico que, hasta cierto punto, ha dado al idealismo trascendental un sentido, quizá, más preciso.

2. Como he intentado hacer ver: hay un carácter histórico que posee la postura de Kant, que resulta ineludible para un estudio completo de su filosofía Desde Untersuchung, donde Kant ponía el fundamento del conocimiento de lo moral en el sentimiento, hasta la Fundamentación donde se lo sitúa en la inteligencia dista un largo camino. Éste no ha sido suficientemente estudiado aquí, pero sí que se ha podido ver que la Dissertatio y la luz del 69 constituyen la piedra de toque hacia el criticismo. De esto se sigue que el modo en el que se interprete dicho evento y dicha obra contribuye en gran medida a lograr una comprensión distinta del sistema crítico en toda su complejidad.

3. La espontaneidad con la que se plantea el deber en Kant se debe a que ésta es una característica fundamental de los conceptos intelectuales, que se oponen a los empíricos.

\section{Bibliografía.}

a)Fuentes primarias:

KANT, I., Lecciones de ética, trad. Roberto Rodríguez Aramayo y Concha Roldán Panadero, Editorial Crítica, Barcelona, 1988

- "Sobre la nitidez de los principios de la teología natural y la moral", trad. de Roberto Torretti, Diálogos, 27, (1974), pp. 57-87

- Sobre la forma y los principios del mundo sensible y del inteligible, trad. Ramón Ceñal, Consejo superior de investigaciones científicas, Madrid, 1961. 
- Del primer fundamento de la primera diferencia de regiones en el espacio, en: Olesti, J., Kant y Leibniz: la incongruencia en el espacio, UPV, Valencia, 2004, pp. $110-118$.

- Handschriftlicher Nachla 4 en: Kant's gesammelte Schriften, Ak. 17 Preussichen Akademia der Wissenschaften, Berlin, de Gruyter, 1902-.

b) Fuentes secundarias:

CARL, W., Der schweigende Kant. Die Entwürfe zu einer Deduktion der Kategorien vor 1781, Vandehoeck \& Ruprecht, Göttingen, 1989

LLANO, A., Fenómeno y Trascendencia en Kant, $2^{\mathrm{a}}$ ed., EUNSA, Pamplona, 1973.

FALGUERAS, I., Perplejidad y filosofía trascendental en Kant, Cuadernos de Anuario filosófico, Serie universitaria $\mathrm{n}^{\circ} 71$. Servicio de Publicaciones de la Universidad de Navarra, Pamplona, 1999.

TONELLI, G., "Die Umwälzung von 1769 bei Kant" en: Kant Studien, 54, (1963), p. 369-375.

VILLACANAS, J.L., La formación de la Crítica de la razón pura, Universidad de Valencia, Valencia, 1980.

RAFAEL REYNA FORTES es doctorando en filosofía en la Universidad de Navarra.

Lineas de investigación:

Filosofía trascendental: Kant y Polo

Publicaciones recientes:

"La libertad posible", en GINOCCHIO-ZORROZA (eds.), Estudios sobre la libertad en la filosofía de Leonardo Polo, Cuadernos de anuario filosófico 247 (2012), pp. 9-12.

Dirección electrónica: rafaelreynafortes@gmail.com

Claridades. Revista de filosofía 7 (2015) 